\title{
Verschwörungstheoretische Medienkritik
}

\author{
Wächter oder Totengräber der Demokratie? - Plädoyer für \\ einen neuen Realismus in der Medienkritik. Von Patrick Zoll
}

Patrick Zoll SJ, M.A. ist Promovend an der Universität Bonn und Lehrbeauftragter für Politische Philosophie

und Sozialethik an der Hochschule für Philosophie in München.
Abstract Der Kampfbegriff Lügenpresse erschüttert seit einiger Zeit die Medienlandschaft. Bei dem mit diesem Wort verknüpften Vorwurf geht es nicht mehr um die Kritik an einem einzelnen Text, einem Bild oder Video, sondern ganz grundsätzlich um die Glaubwürdigkeit des in den Leitmedien Dargestellten und Berichteten. Dem Journalismus wird in pauschaler und oft irrational anmutender Weise sein Anspruch auf Wahrheit abgesprochen und ein manipulatives Machtinteresse unterstellt. Wie kann und sollte man aus medienethischer Sicht auf solche und andere verschwörungstheoretische Vorwürfe reagieren? Für einen angemessenen und effektiven Umgang mit derartigen Infragestellungen von Glaubwürdigkeit ist es erforderlich, zwei Typen von Verschwörungstheorien mit unterschiedlichem Rationalitätspotential zu unterscheiden, deren Genese eng mit der Entstehung und Radikalisierung einer postmodernen Erkenntniskritik verknüpft ist. Diese Analyse führt zu einem neuen Realismus, mittels dessen eine normative Einrahmung der Medienkritik möglich ist, die hilft zu unterscheiden, wo und welche Medienkritik gerechtfertigt ist.

\section{Zwei Typen von Verschwörungstheorien}

$\mathrm{Zu}$ beginnen ist mit einer Phänomenbeschreibung, gemäß derer sich zwei Typen von Verschwörungstheorien anhand eines funktionalen Kriteriums unterscheiden lassen. Der erste Typ hat primär einen kritisch-revelatorischen Anspruch: Er will ein konspiratives und manipulatives Zusammenspiel mächtiger Akteure aufdecken. Als jüngere Beispiele könnte man hier die Enthüllungen rund um den NSA- oder NSU-Skandal anführen oder die Offenlegung der jahrelangen Vertuschungen von sexueller Gewalt in kirchlichen und reformpädagogischen Institutionen, womit zugleich schon deutlich wird, dass man nicht jede zunächst verschwörungstheoretisch anmutende These einfach ungeprüft als Spinnerei abtun sollte. Auch wenn Verschwörungstheorien dieses Typs auf den ers- 
ten Blick als unwahrscheinliche und unplausible Erklärungen gegenüber etablierten Erklärungen für geschichtliche Ereignisse und Zusammenhänge erscheinen, so haben sie doch ein Rationalitätspotential.

Neben diesem gnostischen Typ scheint im zeitgenössischen Zusammenhang aber ein antagonistisches Modell von Verschwörungstheorien dominierend, dessen Funktionsweise sich am Beispiel des Vorwurfs der „Lügenpresse“ gut veranschaulichen lässt. Bekanntermaßen sind diese Wortschöpfung und der damit verbundene Vorwurf weder neu, noch besonders originell. Es handelt sich hierbei um eine rhetorische bzw. argumentative Stilfigur, die seit der Mitte des 19. Jahrhunderts von Anhängern verschiedenster Weltanschauungen verwendet wird, um im Kontext von kulturellen oder politischen Machtkämpfen die mediale Berichterstattung zu diskreditieren, die nicht dem eigenen Machtanspruch dient oder ihm sogar kritisch widerspricht. Auf dieses Mittel wird zurückgegriffen, um die Glaubwürdigkeit der Gegenseite als verlässliche Quelle von Wissen zu unterminieren. Die Wirksamkeit dieses strategischen Mittels hängt dabei entscheidend davon ab, wie gut es dem Kritiker gelingt zu verschleiern, dass er selbst mit dem Vorwurf der Lügenpresse in den angeprangerten Machtkampf mit den Mitteln des Machtkampfs einsteigt.

Wie der Begriff Lüge deutlich macht, glückt diese Verschleierung in dem Maße, in dem es gelingt, sich mit dem Gewand der Wahrheit bzw. des Wissens zu bedecken, um so selbst nicht als Kämpfer für die eigenen Interessen und Machtbestrebungen zu erscheinen, sondern vielmehr als Vertreter und Befreier der Wahrheit. Diese auch als Desinformations-Kampagne bekannte Strategie kommt derzeit offenbar massiv in russischen Medien zum Einsatz, was in Bezug auf die Krim-Invasion nun auch präsidial bestätigt ist. Bei genauerer Betrachtung handelt es sich bei dem problematischen antagonistischen Typ um eine parasitäre Form einer gnostischen Verschwörungstheorie. Sie kann als parasitär bezeichnet werden, weil sie sich nur einen kritischrevelatorischen Anschein gibt, um die Durchsetzung ihrer eigenen Machtinteressen als kritischen Dienst an Wahrheit und Wissen zu tarnen. Man sät Zweifel nicht, um Wissen und Wahrheit zu ernten, sondern will vernebeln, um möglichst unentdeckt einen strategischen Vorteil in einem Machtkampf zu erzielen. Verschwörungstheorien dieses Typs sind deshalb ohne Rationalitätspotential. Es gibt keine unabhängigen und objektiven Fakten mit denen ihre Plausibilität erschüttert werden 
könnte, keine interessensunabhängigen Rationalitätsstandards anhand derer ihre Vernünftigkeit gemessen werden könnte. Für einen möglichst geschickten Umgang mit ihnen sollte die Medienethik sich deshalb weniger von der Philosophie, als von der Psychologie Rat holen. ${ }^{1}$

\section{Erkenntniskritik im Dienste einer Machtkritik}

Allein durch die Unterscheidung dieser beiden Typen von Verschwörungstheorien ist aber noch nicht viel gewonnen. Ausstehend ist noch eine Erklärung, die verständlich macht, warum verschwörungstheoretische Vorwürfe antagonistischen Typs trotz ihres mangelnden Rationalitätspotentials derzeit so erfolgreich sein können, dass sie das Grundvertrauen in den Wahrheitsgehalt und -anspruch medialer Berichterstattung erschüttern können. Im Folgenden wird deshalb ein philosophischer Erklärungsversuch entwickelt, der besagt, dass dieser Erfolg nur verständlich wird, wenn man ihn in den Kontext eines größeren philosophischen bzw. erkenntnistheoretischen Klimawandels einbettet. Hier wird die These vertreten, dass die skizzierten Typen von Verschwörungstheorien ihre philosophischen Wurzeln in einer berechtigten Erkenntnis- und Sozialkritik der Postmoderne haben, deren Erfolg bzw. Pauschalisierung aber zu einem relativistischen Nebel geführt hat, in dem eine postmoderne Sozialkritik ihre instrumentelle Indienstnahme für Machtinteressen nicht mehr kritisch hinterfragen kann, weil ihr der dazu nötige Realismus fehlt.

Die Erzählung über den angedeuteten philosophischen Klimawandel kann man im 19. Jahrhundert mit Friedrich Nietzsche beginnen, weil dieser mit seiner genealogischen Methode ${ }^{2}$ und damit verbundenen Ablehnung moderner bzw. aufgeklärter Erkenntnis- und Rationalitätsideale als der Urvater einer postmodernen Kritik an Wissens- und Wahrheitsansprüchen gelten kann. ${ }^{3}$ Worauf Maurizio Ferraris richtig hinweist, ist, dass sich die Attraktivität und Kraft dieser philosophischen

1 Eine vertiefende Analyse mit praktischen Tipps hierfür bietet Herrmann 2013.

2 Gemeint ist hiermit vor allem die Vorgehensweise, die Nietzsche in den Schriften "Jenseits von Gut und Böse" und "Zur Genealogie der Moral" entwickelt (vgl. Nietzsche 1988).

3 Mehr zu dieser Methode und der philosophiegeschichtlichen Verortung und Interpretation von Nietzsche findet sich bei MacIntyre 1982, S. 21-22, 110, 256-263; 2006, S. 155-166; 1990, S. 32-57, 196-216. 
Bewegung nicht primär aus ihren erkenntnistheoretischen bzw. skeptischen Thesen ableitet, sondern vielmehr daraus, dass sie diese Thesen in den Dienst eines emanzipatorischen und befreienden Pathos stellt (vgl. Ferraris 2014, S. 17, 26), welcher interessanterweise das kritisch-befreiende Moment der Aufklärung unter negativem Vorzeichen bewahrt. Diese emanzipatorische Verheißung kann mit den Schlagwörtern Entsublimierung, Entobjektivierung und Ironisierung charakterisiert werden. ${ }^{4}$ Ausgangspunkt dieses von Nietzsche initiierten philosophischen Programms ist die Idee einer erforderlichen Entsublimierung, was meint, dass es eben nicht - wie die Aufklärung irrtümlich annahm -, die Vernunft ist, die den Menschen aus seiner selbstverschuldeten Unmündigkeit befreit, sondern vielmehr die Anerkennung des eigenen Wollens und Begehrens. Die kritische und emanzipatorische Aufgabe einer postmodernen Sozialkritik besteht deshalb vornehmlich in einer Entobjektivierung, die jeden Rekurs auf Wahrheit und Wissen als verschleierten dogma-

Postmoderne Sozialkritik besteht hauptsächlich in einer ironischen Dekonstruktion von Wirklichkeitskonstruktionen. tischen Machtgestus entlarvt; dieser hat in Wirklichkeit die manipulative Funktion, das eigene subjektive Wollen durch den Hinweis auf vermeintlich anzuerkennende objektive Fakten, Gründe oder Tatsachen zu verbergen. ${ }^{5} \mathrm{Um}$ dies zu erreichen, ist eine Ironisierung geboten, die Distanz zu jeglichen Wahrheits- und Wissensansprüchen schafft und durch diese Distanzierung zu Bewusstsein bringt, dass man es nie mit den Dingen an sich bzw. Tatsachen, sondern nur mit Interpretationen, Vermittlungen und Zeichen zu tun hat. Postmoderne Sozialkritik besteht demnach hauptsächlich in einer ironischen Dekonstruktion von Wirklichkeitskonstruktionen (vgl. Ferraris 2014, S. 18).

Sozialkritik in diesem postmodernen bzw. konstruktivistischen Sinne ist also vornehmlich Erkenntniskritik im Dienste

4 Diese Begriffe übernehme ich mit einer leicht modifizierten Bedeutung von Ferraris, an dessen Ausführungen und Ideen ich mich in der folgenden Charakterisierung einer konstruktivistischen Sozialkritik lose anlehne (vgl. Ferraris 2014, S. 17-27).

5 Philosophiegeschichtlich erscheint mir deshalb MacIntyres Analyse zutreffend, dass die Genese postmoderner emotivistischer bzw. nonkognitivistischer Positionen verstanden werden muss als Reaktion auf das Scheitern G. E. Moores, mittels eines Intuitionismus die objektive Geltung von moralischen Urteilen zu rechtfertigen (vgl. MacIntyre 1982, S. 16-18, 72). 
einer Machtkritik. Vor dem Hintergrund der philosophiegeschichtlichen Skizze dieses postmodernen Emanzipationsprojekts sind gnostische Verschwörungstheorien also gar nichts Ungewöhnliches, sondern werden als radikale Spielarten oder Auswüchse einer allgemeineren Form von Sozialkritik verständlich. Selbst pauschalisierenden Vorwürfen wie dem der Lügenpresse kann von einer solchen Interpretation her also zunächst einmal das Potential zugesprochen werden, eine wichtige medienethische Korrekturfunktion auszuüben: Sie stellen das durch die Leitmedien etablierte domi-

Selbst pauschalisierenden Vorwürfen wie dem der Lügenpresse kann das Potential zugesprochen werden, eine medienethische Korrekturfunktion auszuüben. nante Narrativ in Frage; sie kritisieren die Auswahl der Ereignisse, über die berichtet wird, oder die Bilder, mit denen diese Ereignisse illustriert und interpretiert werden. Screcko Horvat fasst diese Einsicht der Postmoderne ganz gut zusammen, wenn er im Zusammenhang mit der so genannten Finger-Affäre um Yanis Varoufakis schreibt: „Zeichen sind Waffen; jeder Kampf ist auch ein semantischer, das heißt ein Kampf um die Deutungshoheit von Zeichen. Hier gilt die Antwort, die Humpty Dumpty auf Alice Frage: Wie kannst du einem Wort so viele verschiedene Bedeutungen geben?', lieferte: ,Die Frage ist, wer die Macht hat, das ist alles'“ (Horvat 2015, S.11).

\section{Durch die Radikalisierung der Erkenntniskritik zur Paranoia}

Horvats Hinweis auf Carrolls Figur des Humpty Dumpty aus "Alice hinter den Spiegeln“ illustriert aber ungewollt auch das Scheitern des postmodernen Emanzipationsprojekts, das darin begründet liegt, dass die Erkenntniskritik der Postmoderne sich so radikalisiert hat, dass sie nicht mehr in der Lage ist, eine effektive Machtkritik auszuüben und damit ihr emanzipatorisches Erbe verrät. Denn mit der Radikalisierung des Zweifels hin zu einem umfassenden Konstruktivismus bzw. Relativismus verschwindet auch das Rationalitätspotential von Verschwörungstheorien als ernstzunehmenden und selbst kritisierbaren alternativen Erklärungen der Wirklichkeit.

Wenn es - wie bei Alice - unmöglich geworden ist, zwischen Traum beziehungsweise Konstruktion und Wirklichkeit zu unterscheiden, dann kann man Machtausübung auch nicht mehr mit einem Rekurs auf Wahrheit, Wissen oder Fakten kritisieren, denn hierauf wird der kritisierte Mächtige nur das postmoderne Credo rezitieren: „Es gibt keine Tatsachen, nur 
Interpretationen“" ${ }^{6}$. Schlimmer noch: Der Mächtige kann die als Machtkritik gedachte Erkenntniskritik instrumentalisieren, um die Evidenz mit der sein Machtgebrauch kritisiert wird, als konstruiert und verfälscht zu diskreditieren und genealogisch als lediglich konkurrierendes Narrativ delegitimieren, welches eigene Machtansprüche verdeckt?? Der skeptische Zweifel verliert seine methodisch-emanzipative Funktion und die für Descartes zu diesem Zweck bloß durchdachte Fantasie eines nahezu allmächtigen Täuschers wird in der vollendeten Postmoderne als ernstzunehmende Deutung der Wirklichkeit erwogen. Es sollte daher nicht verwundern, dass in einem solchen paranoiafreundlichen Klima Verschwörungstheorien antagonistischen Typs eine neue Konjunktur erleben. Wenn sowohl die grundlegende Unterscheidung zwischen Traum und Wirklichkeit fraglich wird als auch jegliches Vertrauen in verlässliche epistemische Autoritäten ${ }^{8}$ verloren geht und genuines Wissen nur noch durch die mysterienhafte Initiation in die richtig informierten Kreise gewonnen werden kann, dann ist die Paranoia ironischerweise als rationale Reaktion auf diesen Zustand anzusehen.

\section{Plädoyer für einen neuen Realismus}

Die vorausgehenden Überlegungen lassen sich nun abschließend fruchtbar machen für die Entwicklung einer Kriteriologie mittels derer entschieden werden kann, ob und inwieweit eine Medienkritik, die sich verschwörungstheoretisch geriert, als berechtigt oder unberechtigt angesehen werden kann. Das entscheidende Kriterium ist hier die Frage nach dem Rationalitätspotential. Beruft sich die Medienkritik auf eine Verschwö-

6 Ferraris bezeichnet diese Überzeugung als zentralen Glaubenssatz bzw. Dogma der Postmoderne, der sich als programmatische Ankündigung schon bei Nietzsche findet (vgl. Ferraris 2014, S. 16).

7 Diese Indienstnahme eines Skeptizismus für die Politik der Herrschenden als Ergebnis einer pauschalisierten postmodernen Erkenntniskritik wird von Ferraris als Realytismus bezeichnet und treffend mit Bezügen zur Politik- und Medienwelt der Gegenwart näher illustriert (vgl. Ferraris 2014, S. 26-29).

8 Jüngst hat Linda Zagzebski eine interessante Studie zu diesem Thema vorgelegt und dafür argumentiert, dass eine Rehabilitierung von Konzepten wie Autorität und Vertrauen in der Erkenntnistheorie nicht notwendigerweise mit Konzepten wie Autonomie in Widerspruch stehen muss (vgl. Zagzebski 2012). 
rungstheorie gnostischen oder antagonistischen Typs? Wenn sie sich klar dem letztgenannten Typ zuordnen lässt, dann besitzt sie kein Rationalitätspotential und eine Kritik der Medienkritik, die meint, eine rationale Widerlegung der entsprechenden Mythen wäre angesagt, muss zwangsläufig ins Leere laufen. Wie z. B. Sebastian Herrmann im Rückgriff auf zahlreiche empirische psychologische Studien herausgearbeitet hat, lassen sich verschwörungstheoretische Mythen ohne Rationalitätspotential (z. B. Leugnung des Klimawandels, Leugnung der Existenz von AIDS oder Masern etc.) nicht mit Rekurs

Die Medienethik sollte in einem paranoiden kulturellen Klima ihre Aufgabe der Beruhigung und Vertrauensbildung nicht unterschätzen. auf Fakten, Informationen oder Logik entkräften (Herrmann 2013). ${ }^{9}$ Aufgabe der Medien ist dann nicht primär die Aufdeckung von Lügen oder die Verteidigung des eigenen Wahrheitsanspruchs, sondern vielmehr die

Frage, wie das Behauptete möglichst so dargestellt wird, dass es sich auch wahr anfühlt. ${ }^{10}$ Eine Medienethik sollte in einem paranoiden kulturellen Klima insofern ihre Aufgabe der Beruhigung und Vertrauensbildung nicht unterschätzen. ${ }^{11}$

Wie kann man aber entscheiden, ob eine Verschwörungstheorie Rationalitätspotential hat oder nicht? Es zeigt sich an dieser Stelle, dass die Medienethik einer verstärkten metaphilosophischen Reflexion ihrer erkenntnistheoretischen Grundlagen bedarf, die sie dann auch in die Lage versetzt, die vorgetragene Medienkritik ihrerseits kritisch mit ihren epistemologischen

9 Ferraris spricht in diesem Zusammenhang auch von einer pejorativ gemeinten Wiederkehr des Mythos bzw. einer neuen Mythologie (Ferraris 2014, S. 25).

10 Neben Herrmann argumentiert auch Jonathan Haidt im Rückgriff auf Daniel Kahneman und seine Studien zur empirischen Psychologie überzeugend dafür, dass das Problem im Umgang mit Verschwörungstheoretikern oder Fundamentalisten oftmals darin besteht, dass Verschwörungstheorien simplifizieren und sich deshalb einfach wahrer anfühlen als komplexere wissenschaftliche Erklärungen (vgl. Haidt 2012, Kahneman 2011). Vor diesem Hintergrund könnte man eine verschwörungstheoretische Medienkritik insofern auch als Indikator für eine Kommunikationskrise zwischen den Leitmedien und einer Teilöffentlichkeit interpretieren.

11 Eine wichtige vertrauensbildende Maßnahme wird hier die Etablierung bzw. der Ausbau einer Fehlerkultur sein, in der die Medien auf eigene Fehler in der Berichterstattung hinweisen und diese korrigieren. Jüngst hat z. B. das ZDF auf seiner Website nach dem Vorbild der "New York Times“ eine solche Rubrik eingeführt (vgl. Frey 2015, bzw. http://www.heute.del zdf-in-eigener-sache-korrekturen-37527168.html). 
Prämissen und Hintergrundannahmen zu konfrontieren. Weil die Postmoderne mit ihrem Abschied von der Wahrheit und der Radikalisierung ihrer Erkenntniskritik als Teil des Problems $\mathrm{zu}$ betrachten ist, erscheint ein neuer Realismus notwendig, der nicht pauschal das kritische und emanzipatorische postmoderne Anliegen einer Erkenntniskritik im Dienste einer Machtkritik verwirft ${ }^{12}$, aber doch die Radikalisierung dieses Programms in den Thesen, dass alle Tatsachen nur Interpretationen sind, jedes Feststellen ein Akzeptieren darstellt und jeglicher Wissensanspruch ein verdeckter Machtanspruch ist, als Trugschlüsse entlarvt. ${ }^{13}$

Da diese Diskussion hier nicht geführt werden kann, sollen auf der Grundlage eines minimalen Realismus, der behauptet, dass es einen realen Unterschied zwischen Ontologie und Epistemologie bzw. Wahrheit und Rechtfertigung gibt, abschließend einige Überlegungen hinsichtlich des Rationalitätspotentials einer verschwörungstheoretisch formulierten Medienkritik vorgestellt werden. ${ }^{14}$ Folgende Definition für Verschwörungstheorien mit Rationalitätspotential erscheint sinnvoll ${ }^{15}$ :

Bei einer Verschwörungstheorie handelt es sich um ein Erklärungsmodell (EM) mit Rationalitätspotential für ein ge-

12 Neben Ferraris sind hier die Arbeiten von Markus Gabriel zu erwähnen, als einem der wichtigsten deutschen Vertreter eines solchen Neuen Realismus (vgl. Gabriel 2013a, 2015, 2013b).

13 Mehr zu diesen Trugschlüssen und ihrer Kritik findet sich bei Ferraris (2014, S. 33-83).

14 Während der Neue Realismus zur Verteidigung eines solchen minimalen Realismus bei der Unterscheidung zwischen Epistemologie und Ontologie ansetzt, finden sich ähnliche Überlegungen bei einigen neo-pragmatischen Autoren, die dafür argumentieren, dass es einen bleibenden Unterschied zwischen der Wahrheit einer Aussage und ihrer Rechtfertigung gibt und ein Bezug auf Wahrheit eine wichtige Rolle in unseren theoretischen wie praktischen Lebensvollzügen spielt (vgl. Misak 2004, 2000; Stout 2004).

15 Es kann sich hier natürlich nur um einen sehr groben Definitionsversuch handeln, dessen Adäquatheit ich hier nicht eingehender begründen und diskutieren kann. Ich präsentiere also eher einen Vorschlag, der sich in dem Maße als adäquat und brauchbar erweist, wie er in der Praxis hilft, zwischen verschiedenen Typen von Verschwörungstheorien zu unterscheiden und zu erklären, warum ein differenzierter Umgang mit einer verschwörungstheoretischen Medienkritik gerechtfertigt ist. Allerdings sei erwähnt, dass sich dieser Definitionsversuch mitsamt der aus ihm abgeleiteten Kriteriologie mit Ergebnissen konvergiert, die in neueren philosophischen Monographien zu Verschwörungstheorien präsentiert und umfassender gerechtfertigt worden sind (vgl. Dentith 2014; Sunstein 2014). 
schichtliches Ereignis (GE), wenn zugleich folgende Bedingungen erfüllt sind:

(1) Antagonistische Bedingung: Beansprucht, eine alternative Erklärung (EM) für GE zu liefern.

(2) Gnostische Bedingung: Beansprucht, ein etabliertes Konkurrenzmodell (KM) für die Erklärung von GE als falsch und dessen Akzeptanz als Produkt eines konspirativen bzw. manipulativen Zusammenspiels von Akteuren zu entlarven.

(3) Rechtfertigungsbedingung: Beansprucht, die Kritik an $\mathrm{KM}$ zu rechtfertigen und mit EM eine bessere Erklärung als KM für GE zu liefern.

Bedingung eins ist sehr allgemein und greift die genealogische bzw. postmoderne Intuition auf, dass Erkenntniskritik immer auch Machtkritik ist. Anhand ihrer ist es aber noch nicht möglich, klar zwischen rationalen Verschwörungstheorien gnostischen Typs und irrationalen Verschwörungstheorien antagonistischen Typs zu unterscheiden.

Die zweite Bedingung grenzt Verschwörungstheorien nicht nur gegenüber anderen devianten Minderheitstheorien ab, sondern formuliert auch eine realistische Hintergrundannahme einer Erkenntniskritik, die sich im Dienste einer Machtkritik versteht: Es gilt eine vorherrschende Meinung bzw. einen Glauben, der in der Gestalt des Wissens auftritt, als bewusste Täuschung also Pseudo-Wissen zu kritisieren. Dies setzt voraus, dass es Sinn macht, zwischen Glauben und Wissen, Tatsachen und Interpretationen, Sein und Schein bzw. Wahrheit und Täuschung zu unterscheiden. Es wurde bereits skizziert, dass mit dem Verlust solcher realistischer Unterscheidungen in der Postmoderne und der Radikalisierung ihrer Erkenntniskritik auch der Rationalitätsanspruch ihrer Machtkritik verloren geht. Eine verschwörungstheoretische Medienkritik, die die gnostische Bedingung nicht erfüllt, d.h. es für prinzipiell unmöglich hält Wissens- und Wahrheitsansprüche argumentativ einzulösen, muss dementsprechend als irrational und paranoid beurteilt und entsprechend behandelt werden.

Einen letzten Lackmustest für das Rationalitätspotential von verschwörungstheoretischen Vorwürfen stellt schließlich Bedingung drei dar. Sie besagt, dass derjenige, der eine abweichende Erklärung (EM) für ein bestimmtes geschichtliches Ereignis (GE) vorträgt, die eine gut begründete Standarderklärung (KM) kritisch in Frage stellt, eine zweifache Rechtferti- 
gungspflicht übernimmt: Erstens muss er Gründe vortragen, die seine Kritik an der bisher besten Erklärung (KM) für GE plausibilieren, zweitens muss er Gründe präsentieren, die dafür sprechen, dass seine deviante Erklärung (EM) eine bessere Erklärung für GE liefert als KM.

Die Begriffe bessere und beste Erklärung sind normativ zu verstehen und weisen darauf hin, dass das, was eine gute, bessere und beste Erklärung darstellt, nicht einfach der subjektiven Geschmackswahl anheimgestellt ist, sondern die Kriterien dafür durch die jeweilige Forschungspraxis bzw. Forschungsdisziplin festgelegt werden. ${ }^{16}$ Versucht z. B. ein Impfgegner seine Ablehnung einer Impfempfehlung für Masern, die mit Hinweis auf wissenschaftliche Studien zum Impfrisiko und zur Wirksamkeit des Impfstoffes gut begründet ist, damit zu rechtfertigen, dass Masernviren gar nicht existieren, so kann er nicht einfach darauf verweisen, dass diese bisher niemand gesehen hat. Wenn er als Medizinkritiker ernsthaft die Existenz von Masernviren infrage stellen will und damit eine Impfempfehlung delegitimieren will, so muss er danach fragen, wie in der medizinischen Forschung wissenschaftliche Hypothesen begründet und widerlegt werden und welchen Kriterien eine Erklärung genügen muss, um als gut gelten zu dürfen. Dieser normative Aspekt von Rechtfertigung kann vielleicht noch etwas allgemeiner gefasst werden mit der Frage, ob die verschwörungstheoretische Überzeugung und darauf aufbauende Medienkritik nicht nur geschlossen, sondern auch offen gerechtfertigt werden kann. ${ }^{17}$

16 Im Hintergrund stehen hier MacIntyres wissenschaftstheoretische bzw. methodische Überlegungen zu wissenschaftlichem Fortschritt, wonach Rationalitätsstandards und mit ihnen verbundene Normen zur Beurteilung darüber, ob eine Erklärung gut oder schlecht ist, immer praxis- bzw. traditionsimmanent sind, es aber trotzdem möglich ist, verschiedene Praktiken bzw. Traditionen dahingehend zu vergleichen, ob sie bessere oder schlechtere Antworten auf wissenschaftliche Fragen und Probleme geben. Systematisch gesprochen bedeutet dies, anzunehmen, dass es widerspruchsfrei möglich ist, einen epistemischen Kontextualismus mit einem ontologischen Realismus bzw. einem realistischen Wahrheitsbegriff zu verbinden, d.h. die Übernahme eines Kontextualismus nicht notwendigerweise die Akzeptanz eines Relativismus oder Konstruktivismus impliziert. Dies bedürfte einer vertiefenden Ausführung, die hier nicht geleistet werden kann. Es sei hierfür verwiesen auf Lutz 2004, MacIntyre 1977, 1990, 2003, Trenery 2014, Zoll 2010, 2011.

17 Die Begriffe "offene "und "geschlossene Rechtfertigung "übernehme ich von Gaus 1996, S. $130 f f$. 
Gemeint ist damit, dass es nicht ausreicht, allein mit Bezug auf das persönliche Netz von Überzeugungen und Werten in einem geschlossenen Sinne, eine bestimmte Überzeugung für wahr zu halten. Als in einem rationalen Sinne gerechtfertigt kann eine Überzeugung nur gelten, wenn sie offen ist für eine Auseinandersetzung mit Informationen, Überzeugungen und Gründen, die für die Falschheit dieser Überzeugung sprechen, und sie sich in einer solchen dialektischen bzw. dialogischen Auseinandersetzung erfolgreich bewähren kann.

Verschwörungstheoretische Medienkritik ist hilfreich, weil sie vor einer allzu großen Naivität und einem romantischen Realismus bewahrt, gemäß dem das Dargestellte einfach identisch ist mit dem Wirklichen. Sie kann mit ihrem Zweifel

Verschwörungstheoretische

Medienkritik ist hilfreich, weil sie vor einer allzu großen Naivität und einem romantischen Realismus bewahrt. somit eine Art von Wächterfunktion in einer demokratischen Gesellschaft ausüben. Wie in diesem Artikel dargestellt, braucht es aber auch eine normative Einrahmung der Medienkritik, einer Kritik der Medienkritik, die daran erinnert, dass man zwar an allem zweifeln kann, nur es nicht besonders gesund ist, alles zugleich in Zweifel ziehen zu wollen. Alle Kommunikation lebt letztlich auch von Autorität und Vertrauen. Eine verschwörungstheoretisch anmutende Medienkritik, die nachweislich kein Rationalitätspotential aufweist, muss sich deshalb die kritische Frage gefallen lassen, wem mit ihrer irrationalen Infragestellung medialer Autorität und der Zerstörung von Vertrauen in die Glaubwürdigkeit bisher überwiegend verlässlicher medialer Quellen letztlich gedient ist. Findet diese Reflexion nicht statt, werden aus Wächtern der Demokratie ihre Totengräber.

\section{Literatur}

Dentith, Matthew R. X. (2014): The Philosophy of Conspiracy Theories. Houndmills, Hampshire.

Ferraris, Maurizio (2014): Manifest des neuen Realismus. Frankfurt a. M.

Frey, Peter (2015): In eigener Sache. Korrekturen. Erklärung auf heute.de vom 18.3., http://www.heute.de/zdf-in-eigener-sache-korrekturen-37527168. html (zuletzt aufgerufen am 27.5.2015).

Gabriel, Markus (2013a): Die Erkenntnis der Welt: Eine Einführung in die Erkenntnistheorie. Freiburg i. Br..

Gabriel, Markus (2013b): Warum es die Welt nicht gibt. Berlin.

Gabriel, Markus (2015): Fields of sense: A New Realist Ontology. Edinburgh.

Gaus, Gerald F. (1996): Justificatory Liberalism: An Essay on Epistemology and Political Theory. Oxford. 
Haidt, Jonathan (2012): The Righteous Mind: Why Good People Are Divided by Politics and Religion. London.

Herrmann, Sebastian (2013): Starrköpfe überzeugen: Psychotricks für den Umgang mit Verschwörungstheoretikern, Fundamentalisten, Partnern und ihrem Chef. Reinbeck.

Horvat, Srecko (2015): Über Zeichen gehen. In Europa wird über einen Mittelfinger mehr geredet als über die dramatische Krise. In: Süddeutsche Zeitung vom 20.3., S. 11.

Kahneman, Daniel (2011): Thinking, Fast and Slow. London.

Kuhn, Thomas S. (42012): The Structure of Scientific Revolutions. Chicago.

Lutz, Christopher S. (2004): Tradition in the Ethics of Alasdair MacIntyre: Relativism, Thomism, and Philosophy. Lanham, Maryland.

MacIntyre, Alasdair (1977): Epistemological Crises, Dramatic Narrative, and the Philosophy of Science. In: The Monist, 6o. Jg., H. 4, S. 453-472.

MacIntyre, Alasdair (21982): After Virtue: A Study in Moral Theory. London.

MacIntyre, Alasdair (1990): Three Rival Versions of Moral Enquiry. Encyclopaedia, Genealogy, and Tradition. Notre Dame, Indiana.

MacIntyre, Alasdair (32003): Whose Justice? Which Rationality? Notre Dame, Indiana.

MacIntyre, Alasdair (2006): Dependent Rational Animals. Why Human Beings Need the Virtues. Chicago/La Salle, Illinois.

Misak, Cheryl J. (2000): Truth, Politics, Morality: Pragmatism and Deliberation. London.

Misak, Cheryl J. (2004): Truth and the End of Inquiry: A Peircean Account of Truth. Oxford.

Nietzsche, Friedrich W. ('1988): Jenseits von Gut und Böse: Zur Genealogie der Moral. Kritische Studienausgabe. Berlin.

Stout, Jeffrey (2004): Democracy and Tradition. Princeton, New Jersey.

Sunstein, Cass R. (2014): Conspiracy Theories and Other Dangerous Ideas. New York.

Trenery, David (2014): Alasdair MacIntyre, George Lindbeck and the Nature of Tradition. Eugene, Oregon.

Zagzebski, Linda T. (2012): Epistemic Authority: A Theory of Trust, Authority, and Autonomy in Belief. Oxford.

Zoll, Patrick (2010): Ethik ohne Letztbegründung? Zu den nicht-fundamentalistischen Ansätzen von Alasdair MacIntyre und Jeffrey Stout. Würzburg.

Zoll, Patrick (2011): How to Proceed Philosophically? A Critique of Alasdair MacIntyre's Narrative-Historicist Conception of Progress. In: Heythrop Journal, 52. Jg., H. 1, S. 104-112. 